\title{
Reply to JGSU-D-08-00664: Intrapancreatic Accessory Spleen: Deficiency in Diagnosis or Therapeutic Success?
}

\author{
Shuichiro Uchiyama $\cdot$ Kazuo Chijiiwa
}

Received: 26 September 2008 / Accepted: 12 November 2008 / Published online: 3 December 2008

(C) 2008 The Society for Surgery of the Alimentary Tract

We wish to express our appreciation for Dr. Kavic's and Dr. Park's interests in our article "Intrapancreatic accessory spleen (IPAS) mimicking endocrine tumor of the pancreas: case report and review of the literature." Their comment on differential diagnosis between the pancreatic neoplasms and IPAS is attractive. We confirmed that splenic endothelial cells were strongly positive for CD8 in our resected specimen.

We did not perform endoscopic ultrasound (EUS)guided fine needle aspiration biopsy (FNAB) for the tumor of the pancreas tail in our case because IPAS was not considered as the differential diagnosis and we undoubtedly considered the tumor in our case was neuroendocrine tumor. Even if we considered the possibility of IPAS and
EUS-guided FNAB was employed preoperatively, surgical treatment would be selected as long as conclusive diagnosis could not be obtained.

Accuracy of EUS-guided fine needle aspiration (FNA) in the diagnosis of pancreatic neuroendocrine tumor is reported as more than $80 \%$, and EUS-guided FNA is necessary for the diagnosis of hypervascular tumors of the pancreas. As suggested, there is no doubt that we can avoid unnecessary surgery if conclusive diagnosis as IPAS is obtained. This newly reported way for the conclusive diagnosis of IPAS by using immunohistochemical technique of CD8 staining should be widely known, leading to conclusive diagnosis without surgical resection.

S. Uchiyama $\cdot$ K. Chijiiwa $(\square)$

Department of Surgical Oncology and Regulation of Organ

Function, School of Medicine, Miyazaki University,

Miyazaki, Japan

e-mail: kazuochi@med.miyazaki-u.ac.jp 